\title{
Health care staffs' perception of patient safety culture in hospital settings and factors of importance for this
}

\author{
Anna Nordin ${ }^{1 *}$, Kersti Theander ${ }^{1,2}$, Bodil Wilde-Larsson $^{1,3}$, Gun Nordström ${ }^{1,3}$ \\ ${ }^{1}$ Faculty of Health, Science and Technology, Karlstad University, Karlstad, Sweden \\ ${ }^{2}$ Primary Care Research Department, County Council of Värmland, Karlstad, Sweden \\ ${ }^{3}$ Faculty of Public Health, Hedmark University College, Elverum, Norway \\ Email: anna.nordin@kau.se
}

Received 5 November 2013; revised 6 December 2013; accepted 16 December 2013

Copyright (C) 2013 Anna Nordin et al. This is an open access article distributed under the Creative Commons Attribution License, which permits unrestricted use, distribution, and reproduction in any medium, provided the original work is properly cited.

\begin{abstract}
Many hospital patients are affected by adverse events. Managers are important when improving safety. The perception of patient safety culture varies among health care staff. Health care staff $(n=1023)$ working in medical, surgical or mixed medical-surgical health care divisions answered the 51 items (14 dimensions) Swedish Hospital Survey on Patient Safety Culture (S-HSOPSC). Respondents with a managerial function scored higher than non-managers for 11 of 14 dimensions, indicating patient safety culture strengths for a majority of dimensions. Enrolled nurses and staff with experience $>10$ years also scored high for several dimensions. The 12 dimensions and sample characteristics explained $49 \%$ and $26 \%$ of the variance for the outcome dimensions Overall Perceptions of Safety and Frequency of Incident Reporting, respectively. RNs, ENs and physicians have different views on patient safety culture. Hospital Management Support and Organisational Learning is some important factors influencing patient safety culture. Bridging the gap in health care staff's perceptions of safety in order to improve patient safety is of utmost importance. Managers have the responsibility to foster patient safety culture at their workplace and can thus benefit from results when improving safety for patients.
\end{abstract}

Keywords: Patient Safety Culture; Hospital; Management; Organizations; Perceptions

\section{INTRODUCTION}

Adverse events are unintended injuries or complications caused by health care rather than by the patient's disease,

\footnotetext{
${ }^{*}$ Corresponding author.
}

leading to death, disability or prolonged hospital stay [1]. Previous studies in various countries have shown that between three and $17 \%$ of all patients experience one or more adverse events during hospital stay [1-9]. In recent years, health care providers have focused on patient safety as a reaction to adverse events. Patient safety culture guides managers and health care staff in their behavior [10]. A dynamic, conscious definition of patient safety culture is presented by European Network for Patient Safety (EUNetPaS): "An integrated pattern of individual and organizational behavior, based upon shared beliefs and values that continuously seeks to minimize patient harm, which may result from the processes of care delivery" [11, p. 4]. A review reveals that in a safety culture, management is a key element to creating and encouraging safety. Learning is highly valued among all staff, and a willingness to learn from mistakes and to constantly improve performance prevails. Furthermore, the spirit of managers and health care staff is collaborative and relations are open, respectful and flexible. Errors are recognized as system failures rather than individual failures, and at the same time, individuals are responsible for their actions [12]. Studies have shown relations between a strong patient safety culture and patient outcome such as shorter length of stay [13], willingness of treatment error reporting [14], fewer falls among patients [15] and lower rates of in-hospital complications e.g. pneumothorax, infection due to care and postoperative sepsis [16].

When improving safety, measuring patient safety culture is of great importance. Examining health care staff's perception of patient safety culture and aspects influencing patient safety culture represents the first step when managers want to improve health care quality and safety.

There are a variety of instruments to measure patient safety culture $[11,17]$. One such instrument is the Hospital Survey on Patient Safety Culture (HSOPSC), devel- 
oped in the US by the Agency for Healthcare Research and Quality (AHRQ). The HSOPSC measures patient safety culture at unit and hospital level, as well as patient safety culture outcome measures. It has been validated and is reported for use in many European countries [11] and in different contexts [18,19].

Earlier studies among health care staff using the HSOPSC have shown that the unit level dimensions "Teamwork Within Hospital Unit" e.g. [20], "Manager Promoting Safety" e.g. [21], and "Organisational Learning" e.g. [22] were highly scored, indicating strengths in patient safety culture. This was also the case for the hospital level dimensions "Hospital Management Support" e.g. $[23,24]$ and "Teamwork Across Hospital Units" e.g. [25]. On the contrary, "Nonpunitive Response To Error" e.g. [26], "Staffing" e.g. [27] and "Feedback and Communication" e.g. [20] were those unit level dimensions which health care staff scored the lowest. This was also seen for the hospital level dimensions "Teamwork Across Hospital Units" e.g. [21], "Hospital Handoffs and Transitions" e.g. [25] as well as for "Hospital Management Support" e.g. [26]. Regarding the outcome measures, results from earlier studies differ. Health care staff scored high, indicating patient safety culture strengths, for "Frequency of Event Reporting" [28] and for "Overall Perceptions of Safety" e.g. [22]. However, health care staff have also scored low for the same measures e.g. [20,21, 24,25].

Patient safety culture measurement studies have shown differences between staff groups. Managers scored higher patient safety culture values than non-managers [23,29,30]. Registered nurses (RNs) scored higher patient safety culture values than physicians [31,32]. Higher scores were also reported for RNs with longer experience in the current unit compared to those with shorter experience [23,29] and for RNs aged 40 - 49 years compared to those younger than that [29]. Patient safety culture perception has also shown to differ due to context, where respondents working in internal medicine and obstetric units reported higher scores considering communication openness when compared to those in other kinds of units [29].

Analyses of relationships between patient safety culture and measures of safety performance [33] showed an explained variance which varied. Ballangrud et al. [21] reported a model consisting of the 12 original dimensions showing an explained variance of $28 \%$ for "Overall Perception of Safety" and "Frequency of Incident Reporting" respectively. Pfeiffer \& Manser [34] used a modified HSOPSC version consisting of nine of the 12 original dimensions. This model showed an explained variance of 54\% for "Overall Perception of Safety" and $35 \%$ for "Frequency of Incident Reporting" [34]. Also Alahmadi [24] used a modified HSOPSC version con- sisting of seven of the 12 original dimensions. This model showed an explained variance of $32 \%$ for "Overall Perception of Safety" [24].

Several factors are known to be of importance for patient safety culture; e.g. managers' actions and support and health care staff characteristics. Teamwork as well as the organisation's ability to learn and improve is also important. Several studies have been conducted regarding patient safety culture as perceived by different health care staff, and differences between groups have also been reported. Studies have also shown differences regarding variations in potential predictors for patient safety culture. These studies were undertaken in different countries, contexts, and health care systems and often in small samples. An additional European study of patient safety culture and patient safety is not only of national interest, but should also contribute to the collective knowledge regarding patient safety culture. To gain a better understanding of managers' and staff's perception of patient safety culture and the factors potentially of importance for this, these relations need further examination.

The purpose of this paper was to compare managers' and health care staff's perceptions of patient safety culture and to explore factors potentially influencing patient safety culture in hospital settings.

\section{METHODS}

\subsection{Sample and Procedure}

The sample consisted of 2120 health care staff (RNs, $n=$ 1225 , enrolled nurses (ENs), $n=633$ and physicians, $n=$ $262)$ employed in a medical $(n=999)$, surgical $(n=821)$ or mixed medical-surgical $(n=300)$ health care division in a Swedish county council. The medical and the surgical health care divisions, including senior management board, span over two hospitals. The mixed medical-surgical health care division constitutes of staff employed at a third hospital, led by a hospital manager and senior managers. Data was collected in late 2009 by means of a cross-sectional web-survey. It was disseminated through a personally-unique link to each employee who fulfilled the following criteria: being an $\mathrm{RN}, \mathrm{EN}$ or physician working at one of three health care divisions. The respondents had to have an extent of service of 50 percent or more, being employed three months or more and not being on extended sick or parental leave. Written information about the study was sent out via staff newspaper and information was published on the staff intranet. Survey aim, information about voluntary participation and confidentiality was emphasized both at information meetings held at the health care divisions as well as in the e-mail message containing the link to the questionnaire. Names and e-mail addresses of eligible respondents $(\mathrm{N}=2120)$ were obtained from nurse managers 
and personnel officers. These were asked to review the names and addresses thoroughly and if necessary adjust them in order to ensure that questionnaires were sent out to the correct recipients.

\subsection{Questionnaire}

Data was collected using the Swedish version of the HSOPSC; Swedish Hospital Survey on Patient Safety Culture (S-HSOPSC). The S-HSOPSC has been translated into Swedish, adapted by adding two dimensions comprising seven items of importance to patient safety culture within a Swedish health care context, i.e. items about reporting risk, information and support to patients, relatives and health care staff in connection with adverse events [35] and statistically validated for use in Swedish hospital settings [36]. The remaining $(n=44)$ items are the same as in the HSOPSC [33]. In total, the SHSOPSC includes 51 items forming 14 dimensions. Seven dimensions (24 items) deal with unit level aspects of patient safety culture, three dimensions (11 items) deal with hospital level patient safety culture. Two dimensions and one single-item ( 7 items) serve as outcome measures. One single-item serve as descriptive measure regarding number of events reported over 12 months. Most items are being answered using a five-point response scale of agreement ( 1 = "Strongly Disagree" to 5 $=$ "Strongly Agree") or frequency $(1=$ "Never" to $5=$ "Always", or 1 = "Excellent" to 5 = "Failing") where a higher score indicates strengths in patient safety. Two items use a six-point frequency scale from "No Event" to "21 Events or more" or "No Risk" to "21 risks or more". In this study, Cronbach's alphas for the 14 dimensions varied between 0.58 and 0.86 . In addition, a demographic question regarding health care division affiliation was added (Table 1).

\subsection{Ethical Considerations}

Before the study was carried out, the project underwent ethical review and approval by the local ethics committee (Dnr. C 2009/304). In addition, the County Council Manager and managers on each health care division gave their approval of the study. Data was handled according to the Personal Data Act (1988) supervised by the Data Inspection Board. Participation was voluntary and confidential.

\subsection{Data Analysis}

Questionnaires were excluded if no entire section was answered, fewer than half of the items were answered or when all items were answered the same e.g. all 5: s in accordance with developer instructions [33]. For each item, the percentage of positive responses was calculated, i.e. the percentage of respondents who answered the item
Table 1. Characteristics of the sample $(n=1023)$.

\begin{tabular}{|c|c|c|c|}
\hline & Mean (SD) & $\mathrm{n}$ & $\%$ \\
\hline \multicolumn{4}{|l|}{ Sex } \\
\hline Female & & 871 & 85 \\
\hline Male & & 152 & 15 \\
\hline Age & $46.1(10.1)$ & & \\
\hline 0 - 40 years & & 271 & 26 \\
\hline $41-55$ years & & 508 & 50 \\
\hline$\geq 56$ years & & 185 & 18 \\
\hline Missing & & 59 & 6 \\
\hline \multicolumn{4}{|l|}{ Managerial function } \\
\hline Manager & & 51 & 5 \\
\hline Non manager & & 972 & 95 \\
\hline \multicolumn{4}{|l|}{ Staff groups } \\
\hline Registered nurses & & 660 & 64 \\
\hline Enrolled nurses & & 294 & 29 \\
\hline Physicians & & 69 & 7 \\
\hline Total work experience & $22.9(11.2)$ & & \\
\hline $0-5$ years & & 81 & 8 \\
\hline $6-10$ years & & 114 & 11 \\
\hline$\geq 10$ years & & 759 & 74 \\
\hline Missing & & 69 & 7 \\
\hline \multicolumn{4}{|l|}{ Health Care Division } \\
\hline Medical & & 439 & 43 \\
\hline Surgical & & 409 & 40 \\
\hline Mixed medical-surgical & & 175 & 17 \\
\hline
\end{tabular}

by checking "strongly agree" and "agree" or "always" and "most of the time" [33]. The data was summarized in frequencies, percentage (nominal data), mean and (SD) (interval data). Variance due to differences in size between two groups were analysed using Levenes test ( $p>$ 0.05 ). Differences in patient safety culture between two unrelated groups (i.e. managerial function, sex) were analysed using Students T-test. Differences in patient safety culture between three unrelated groups (i.e. staff group, total experience within health care, age, workplace division) were tested using ANOVA. Significant F-levels were followed by post-hoc comparison (Tukey) to analyse differences between the groups [37].

A multiple regression analysis was conducted where all independent variables were entered simultaneously [37]. Dependent variables were the two outcome measures "Frequency of Event Reporting" and "Overall Perceptions of Safety". Independent variables were the unit level, hospital level and Swedish added dimensions. Staff group, health care division, sex, managerial function and total experience within health care also served as independent variables. Internal consistency of the patient sa- 
fety dimensions was calculated using Cronbach's alpha coefficient [37,38]. Statistical analyses were conducted using IBM Statistical Package for Social Sciences (SPSS) Statistics version 19.0. Levels of significance were set at $\mathrm{p}<0.01$.

\section{RESULTS}

In total, 1117 health care staff answered the questionnaire (response rate 53\%). Of these, 1023 (48\%) were valid. RNs constituted the largest group, most of the respondents were above 40 years of age and five percent had a managerial function (Table 1).

\section{Differences between Health Care Staff}

Managers and non-managers

Managers scored higher for 11 out of 14 dimensions compared to non-managers. Differences were distributed at outcome measures, unit level, hospital level and the Swedish added dimensions (Table 2).

Staff groups

ENs scored higher in ten out of 14 dimensions compared to RNs and in six dimensions compared to physicians. RNs scored higher than physicians in three dimensions. Physicians scored higher than RNs in one dimension. Differences were distributed at outcome measures, unit level, hospital level and the Swedish added dimen- sions (Table 3).

Sex

Analyses showed that female $(\mathrm{n}=871)$ respondents reported higher patient safety culture scores than male (n = 152) respondents in four dimensions "Frequency of Event Reporting" (mean $=3.41, \mathrm{SD}=0.85$ vs. mean $=$ $3.16, \mathrm{SD}=0.83, \mathrm{t}=-3.331, \mathrm{p}=0.001)$, "Feedback and Communication About Error" (mean $=3.94, \mathrm{SD}=0.73$ vs. mean $=3.72, \mathrm{SD}=0.77, \mathrm{t}=-3.446, \mathrm{p}=0.001)$, "Hospital Management Support for Patient Safety" $($ mean $=3.10, \mathrm{SD}=0.84$ vs. mean $=2.88, \mathrm{SD}=0.83, \mathrm{t}=$ $-3.059, \mathrm{p}=0.002$ ) and "Hospital Handoffs and Transitions" $($ mean $=3.62 \mathrm{SD}=0.64$ vs. mean $=3.43 \mathrm{SD}=$ $0.67, \mathrm{t}=-3.361, \mathrm{p}=0.001)$. Differences were distributed at outcome measures, unit level and hospital level.

Age

Health care staff aged $\geq 56$ years scored higher for eight out of 14 dimensions compared to those aged $\leq 40$ years and for three out of 14 dimensions compared to those aged between 41 and 55 years. Health care staff aged 41 - 55 scored higher for nine dimensions compared to those aged $\leq 40$ years. Differences were distributed at outcome measures, unit level, hospital level and the Swedish added dimensions (Table 4).

Total work experience within health care

Health care staff with experience $>10$ years scored higher for five out of 14 dimensions compared to those

Table 2. Comparisons of patient safety culture scores between managers and non-managers.

\begin{tabular}{|c|c|c|c|c|}
\hline \multirow{2}{*}{$\begin{array}{l}\text { Patient Safety } \\
\text { Culture dimensions }\end{array}$} & $\begin{array}{c}\text { Managers } \\
(n=51)\end{array}$ & $\begin{array}{l}\text { Non-managers } \\
\quad(n=972)\end{array}$ & \multirow[t]{2}{*}{ t-values } & \multirow[t]{2}{*}{ p-values } \\
\hline & $\mathrm{n} /$ Mean (SD) & n/Mean (SD) & & \\
\hline \multicolumn{5}{|l|}{ Outcome Measures } \\
\hline Frequency of Event Reporting ${ }^{1}$ & $51 / 3.69(0.79)$ & $947 / 3.35(0.85)$ & 2.744 & 0.006 \\
\hline Overall Perceptions of Safety ${ }^{2}$ & $51 / 3.89(0.69)$ & $972 / 3.70(0.75)$ & 1.733 & 0.083 \\
\hline \multicolumn{5}{|l|}{ Unit Level } \\
\hline Manager Promoting safety ${ }^{2}$ & $51 / 4.00(0.64)$ & $970 / 3.79(0.77)$ & 1.940 & 0.053 \\
\hline Organisational Learning ${ }^{2}$ & $51 / 3.92(0.59)$ & $971 / 3.58(0.65)$ & 3.688 & 0.000 \\
\hline Teamwork Within Hospital Units ${ }^{2}$ & $51 / 4.27(0.45)$ & $972 / 4.05(0.54)$ & 2.854 & 0.004 \\
\hline Communication Openness ${ }^{1}$ & $51 / 4.24(0.50)$ & $971 / 3.94(0.65)$ & 3.228 & 0.001 \\
\hline Feedback and Communication ${ }^{1}$ & $51 / 4.16(0.56)$ & $971 / 3.90(0.75)$ & 3.093 & 0.003 \\
\hline Nonpunitive Response To Error ${ }^{2}$ & $51 / 4.27(0.68)$ & $972 / 3.78(0.80)$ & 4.330 & 0.000 \\
\hline Staffing $^{2}$ & $51 / 3.76(0.67)$ & $972 / 3.44(0.68)$ & 3.266 & 0.001 \\
\hline \multicolumn{5}{|l|}{ Hospital Level } \\
\hline Hospital Management Support ${ }^{2}$ & $51 / 3.54(0.80)$ & $962 / 3.04(0.84)$ & 4.110 & 0.000 \\
\hline Teamwork Across Hospital Units ${ }^{2}$ & $51 / 3.70(0.56)$ & $966 / 3.45(0.62)$ & 2.925 & 0.004 \\
\hline Hospital Handoffs and Transitions ${ }^{2}$ & $51 / 3.65(0.67)$ & $958 / 3.59(0.65)$ & .686 & 0.493 \\
\hline \multicolumn{5}{|l|}{ Swedish Added dimensions } \\
\hline Information to Patient/Relatives ${ }^{2}$ & $50 / 3.68(0.72)$ & $902 / 3.38(0.77)$ & 2.673 & 0.008 \\
\hline Information to Staff ${ }^{2}$ & $51 / 4.15(0.51)$ & $919 / 3.63(0.87)$ & 6.643 & 0.000 \\
\hline
\end{tabular}

${ }^{1}$ Response alternative ranging from 1 (Never) to 5 (Always); ${ }^{2}$ Response alternative ranging from 1 (Strongly Disagree) to 5 (Strongly Agree). 
Table 3. Comparisons of patient safety culture scores between different staff groups.

\begin{tabular}{|c|c|c|c|c|c|}
\hline \multirow{3}{*}{$\begin{array}{l}\text { Patient Safety } \\
\text { Culture dimensions }\end{array}$} & \multicolumn{3}{|c|}{ Staff groups } & \multirow{3}{*}{$F(\mathrm{df})$} & \multirow{3}{*}{ p-values } \\
\hline & $\begin{array}{l}\text { Registered } \\
\text { Nurses (1) } \\
(n=660)\end{array}$ & $\begin{array}{l}\text { Enrolled } \\
\text { Nurses (2) } \\
(n=294)\end{array}$ & $\begin{array}{c}\text { Physicians } \\
(3) \\
(n=69)\end{array}$ & & \\
\hline & n/Mean (SD) & n/Mean (SD) & n/Mean (SD) & & \\
\hline \multicolumn{6}{|l|}{ Outcome Measures } \\
\hline $\begin{array}{l}\text { Frequency of } \\
\text { Event Reporting }\end{array}$ & $650 / 3.30(0.82)$ & $281 / 3.62(0.87)$ & $67 / 3.01(0.79)$ & $20.96(2)$ & $\begin{array}{l}2>1(\mathrm{p}=0.000) \\
2>3(\mathrm{p}=0.000)\end{array}$ \\
\hline $\begin{array}{l}\text { Overall Perceptions } \\
\text { of Safety }^{2}\end{array}$ & $660 / 3.67(0.76)$ & 294/3.86(0.68) & $69 / 3.44(0.78)$ & $11.57(2)$ & $\begin{array}{l}2>1(\mathrm{p}=0.001) \\
2>3(\mathrm{p}=0.000)\end{array}$ \\
\hline \multicolumn{6}{|l|}{ Unit Level } \\
\hline $\begin{array}{l}\text { Manager Promoting } \\
\text { safety }^{2}\end{array}$ & $660 / 3.73(0.77)$ & $294 / 3.96(0.72)$ & $67 / 3.70(0.81)$ & $9.66(2)$ & $2>1(p=0.000)$ \\
\hline Organisational Learning $^{2}$ & $659 / 3.55(0.66)$ & $294 / 3.74(0.57)$ & $69 / 3.53(0.72)$ & $9.69(2)$ & $2>1(p=0.000)$ \\
\hline $\begin{array}{l}\text { Teamwork Within } \\
\text { Hospital Units }\end{array}$ & $660 / 4.05(0.53)$ & $294 / 4.11(0.51)$ & $69 / 3.98(0.63)$ & $2.10(2)$ & NS \\
\hline $\begin{array}{l}\text { Communication } \\
\text { Openness }^{1}\end{array}$ & $660 / 3.94(0.65)$ & $294 / 3.96(0.65)$ & $68 / 4.00(0.65)$ & $0.21(2)$ & NS \\
\hline $\begin{array}{l}\text { Feedback and } \\
\text { Communication }^{1}\end{array}$ & $660 / 3.91(0.75)$ & $294 / 4.00(0.69)$ & $68 / 3.60(0.83)$ & $8.16(2)$ & $\begin{array}{l}1>3(\mathrm{p}=0.003) \\
2>3(\mathrm{p}=0.000)\end{array}$ \\
\hline $\begin{array}{l}\text { Nonpunitive Response } \\
\text { To Error }^{2}\end{array}$ & $660 / 3.75(0.80)$ & $294 / 3.93(0.75)$ & $69 / 3.73(0.88)$ & $5.71(2)$ & $2>1(p=0.003)$ \\
\hline Staffing $^{2}$ & $660 / 3.51(0.66)$ & $294 / 3.40(0.68)$ & $69 / 3.25(0.86)$ & $6.06(2)$ & $1>3(\mathrm{p}=0.008)$ \\
\hline \multicolumn{6}{|l|}{ Hospital Level } \\
\hline $\begin{array}{l}\text { Hospital Management } \\
\text { Support }^{2}\end{array}$ & $655 / 2.98(0.82)$ & $289 / 3.31(0.82)$ & $69 / 2.91(0.94)$ & $17.01(2)$ & $\begin{array}{l}2>1(\mathrm{p}=0.000) \\
2>3(\mathrm{p}=0.001)\end{array}$ \\
\hline $\begin{array}{l}\text { Teamwork Across } \\
\text { Hospital Units }\end{array}$ & $658 / 3.43(0.58)$ & $290 / 3.57(0.62)$ & $69 / 3.30(0.81)$ & $8.41(2)$ & $\begin{array}{l}2>1(\mathrm{p}=0.002) \\
2>3(\mathrm{p}=0.003)\end{array}$ \\
\hline Hospital Handoffs and Transitions ${ }^{2}$ & $651 / 3.57(0.64)$ & $289 / 3.74(0.62)$ & $69 / 3.14(0.70)$ & $25.86(2)$ & $\begin{array}{l}1>3(\mathrm{p}=0.000) \\
2>1(\mathrm{p}=0.000) \\
2>3(\mathrm{p}=0.000)\end{array}$ \\
\hline \multicolumn{6}{|l|}{$\begin{array}{l}\text { Swedish Added } \\
\text { dimensions }\end{array}$} \\
\hline $\begin{array}{l}\text { Information to } \\
\text { Patient/Relatives }\end{array}$ & $610 / 3.26(0.78)$ & $275 / 3.65(0.69)$ & $67 / 3.63(0.68)$ & $28.94(2)$ & $\begin{array}{l}2>1(\mathrm{p}=0.000) \\
3>1(\mathrm{p}=0.000)\end{array}$ \\
\hline Information to Staff ${ }^{2}$ & $628 / 3.56(0.89)$ & $277 / 3.89(0.75)$ & $65 / 3.60(0.84)$ & $14.34(2)$ & $2>1(p=0.000)$ \\
\hline
\end{tabular}

${ }^{1}$ Response alternative ranging from 1 (Never) to 5 (Always); ${ }^{2}$ Response alternative ranging from 1 (Strongly Disagree) to 5 (Strongly Agree).

with experience 0 - 5 years and for four out of 14 dimensions compare to those with total experience of $6-10$ years. Differences were distributed at outcome measures, unit level, hospital level and the Swedish added dimensions (Table 5).

Health care division

Health care staff working in the mixed medical-surgical health care division reported higher patient safety culture scores for two dimensions compared with those working in the medical and surgical health care division: "Hospital Management Support for Patient Safety" (Mixed: mean $=3.53, \mathrm{SD}=0.80$ vs. Medical: mean $=$ 2.91, $\mathrm{SD}=0.82, \mathrm{p}=0.000$ and Mixed vs. Surgical: mean $=3.04, \mathrm{SD}=0.81, \mathrm{p}=0.000, \mathrm{~F}=36.553)$ and "Teamwork Across Hospital Units" (Mixed: mean $=3.75, \mathrm{SD}=$ 0.63 , vs. Medical: mean $=3.40, \mathrm{SD}=0.57, \mathrm{p}=0.000$ and Mixed vs. Surgical: mean $=3.40, \mathrm{SD}=0.62, \mathrm{p}=0.000, \mathrm{~F}$ $=24.648$ ). Health care staff working in the mixed medi- cal-surgical health care division reported higher patient safety culture scores compared with those working in the surgical health care division for the dimension "Hospital Handoffs and Transitions" (Mixed: mean $=3.77, \mathrm{SD}=$ 0.64 vs. Surgical: mean $=3.50, \mathrm{SD}=0.67, \mathrm{p}=0.000, \mathrm{~F}=$ 10.038) and higher patient safety culture than those working in the medical health care division for the dimension "Information to Patients/Relatives" (Mixed: mean $=3.59 \mathrm{SD}=0.71$ vs. Medical: mean $=3.31 \mathrm{SD}=$ $0.79, \mathrm{p}=0.000, \mathrm{~F}=7.753$ ). Differences were reported at hospital level and the Swedish added dimensions.

Factors influencing patient safety culture

The multiple regression analysis revealed that the patient safety culture dimensions and sample characteristics, i.e. staff group, health care division, sex, managerial function and total experience within health care (Table 6) explained $49 \%$ of the variance for "Overall Perceptions of Safety" and $26 \%$ of the variance for "Frequency of 
Table 4. Comparisons of patient safety culture scores between different age groups.

\begin{tabular}{|c|c|c|c|c|c|}
\hline \multirow{3}{*}{$\begin{array}{l}\text { Patient Safety } \\
\text { Culture dimensions }\end{array}$} & \multicolumn{3}{|c|}{ Age } & \multirow{3}{*}{$F(\mathrm{df})$} & \multirow{3}{*}{ p-values } \\
\hline & $\begin{array}{c}\leq 40 \text { years } \\
\quad(1) \\
(n=271)\end{array}$ & $\begin{array}{c}41-55 \text { years } \\
(2) \\
(n=508)\end{array}$ & $\begin{array}{c}\geq 56 \text { years } \\
\quad(3) \\
(n=185)\end{array}$ & & \\
\hline & n/Mean (SD) & n/Mean (SD) & n/Mean (SD) & & \\
\hline \multicolumn{6}{|l|}{ Outcome Measures } \\
\hline $\begin{array}{l}\text { Frequency of } \\
\text { Event Reporting }\end{array}$ & $267 / 3.10(0.86)$ & $497 / 3.45(0.80)$ & $177 / 3.47(0.85)$ & $18.231(2)$ & $\begin{array}{l}2>1(\mathrm{p}=0.000) \\
3>1(\mathrm{p}=0.000)\end{array}$ \\
\hline $\begin{array}{l}\text { Overall Perceptions } \\
\text { of Safety }^{2}\end{array}$ & $271 / 3.46(0.77)$ & $508 / 3.76(0.72)$ & $185 / 3.95(0.70)$ & $27.724(2)$ & $\begin{array}{l}2>1(p=0.000) \\
3>1(p=0.000) \\
3>2(p=0.007)\end{array}$ \\
\hline \multicolumn{6}{|l|}{ Unit Level } \\
\hline $\begin{array}{l}\text { Manager Promoting } \\
\text { safety }^{2}\end{array}$ & $271 / 3.76(0.76)$ & $507 / 3.79(0.78)$ & $185 / 3.88(0.73)$ & $1.508(2)$ & NS \\
\hline Organisational Learning $^{2}$ & $271 / 3.47(0.70)$ & $507 / 3.62(0.64)$ & $185 / 3.74(0.58)$ & $10.717(2)$ & $\begin{array}{l}2>1(p=0.004) \\
3>1(p=0.000)\end{array}$ \\
\hline $\begin{array}{l}\text { Teamwork Within } \\
\text { Hospital Units }\end{array}$ & $271 / 4.06(0.54)$ & $508 / 4.07(0.52)$ & $185 / 4.05(0.57)$ & $0.209(2)$ & NS \\
\hline $\begin{array}{l}\text { Communication } \\
\text { Openness }^{1}\end{array}$ & $271 / 3.89(0.68)$ & $507 / 3.98(0.63)$ & $185 / 3.98(0.64)$ & $2.011(2)$ & NS \\
\hline $\begin{array}{l}\text { Feedback and } \\
\text { Communication }^{1}\end{array}$ & $271 / 3.82(0.77)$ & $507 / 3.95(0.72)$ & $185 / 3.94(0.76)$ & $3.028(2)$ & NS \\
\hline $\begin{array}{l}\text { Nonpunitive Response } \\
\text { To Error }^{2}\end{array}$ & $271 / 3.68(0.80)$ & $508 / 3.86(0.78)$ & $185 / 3.82(0.85)$ & $4.322(2)$ & $2>1(\mathrm{p}=0.010)$ \\
\hline Staffing $^{2}$ & $271 / 3.33(0.69)$ & $508 / 3.50(0.68)$ & $185 / 3.59(0.69)$ & $9.325(2)$ & $\begin{array}{l}2>1(p=0.002) \\
3>1(p=0.000)\end{array}$ \\
\hline \multicolumn{6}{|l|}{ Hospital Level } \\
\hline $\begin{array}{l}\text { Hospital Management } \\
\text { Support }^{2}\end{array}$ & $268 / 2.75(0.76)$ & $503 / 3.12(0.81)$ & $183 / 3.39(0.85)$ & $37.611(2)$ & $\begin{array}{l}2>1(\mathrm{p}=0.000) \\
3>1(\mathrm{p}=0.000) \\
3>2(\mathrm{p}=0.000)\end{array}$ \\
\hline $\begin{array}{l}\text { Teamwork Across } \\
\text { Hospital Units }\end{array}$ & $270 / 3.30(0.64)$ & $508 / 3.49(0.58)$ & $180 / 3.59(0.63)$ & $14.863(2)$ & $\begin{array}{l}2>1(p=0.000) \\
3>1(p=0.000)\end{array}$ \\
\hline $\begin{array}{l}\text { Hospital Handoffs } \\
\text { and Transitions }{ }^{2}\end{array}$ & $269 / 3.53(0.66)$ & $502 / 3.61(0.63)$ & $179 / 3.64(0.67)$ & $2.012(2)$ & NS \\
\hline \multicolumn{6}{|l|}{$\begin{array}{l}\text { Swedish Added } \\
\text { dimensions }\end{array}$} \\
\hline $\begin{array}{l}\text { Information to } \\
\text { Patient/Relatives }\end{array}$ & $258 / 3.19(0.81)$ & $479 / 3.40(0.75)$ & $168 / 3.67(0.68)$ & $19.953(2)$ & $\begin{array}{l}2>1(p=0.001) \\
3>1(p=0.000) \\
3>2(p=0.000)\end{array}$ \\
\hline Information to Staff ${ }^{2}$ & $265 / 3.42(0.95)$ & $487 / 3.73(0.83)$ & $176 / 3.83(0.77)$ & $15.235(2)$ & $\begin{array}{l}2>1(\mathrm{p}=0.000) \\
3>1(\mathrm{p}=0.000)\end{array}$ \\
\hline
\end{tabular}

${ }^{1}$ Response alternative ranging from 1 (Never) to 5 (Always); ${ }^{2}$ Response alternative ranging from 1 (Strongly Disagree) to 5 (Strongly Agree).

Incident Reporting" (Table 6). The model shows that three unit level dimensions; "Organisational Learning", "Nonpunitive Response To Error" and "Staffing", as well as the hospital level dimensions "Hospital Management Support" and "Hospital Handoffs and Transitions" made significant contributions to "Overall Perceptions of Safety". Two unit level dimensions; "Organisational Learning" and "Feedback and Communication" and one of the Swedish added dimensions; "Information to Patient/Relatives" contributed significantly to "Frequency of Incident Reporting". Concerning the characteristics, managerial function as well as total work experience within health care $>10$ years contributed significantly to "Overall Perceptions of Safety".

\section{DISCUSSION}

The main results of this study show that perception of patient safety culture differed in relation to managerial function, staff group, sex, age, total work experience and health care division. The multivariate regression analysis show that $49 \%$ of the variance for "Overall Perception of Safety" and $26 \%$ of the variance for "Frequency of Incident Reporting" were explained by the patient safety 
Table 5. Comparisons of patient safety culture scores between respondents in relation to total work experience within health care.

\begin{tabular}{|c|c|c|c|c|c|}
\hline \multirow{3}{*}{$\begin{array}{l}\text { Patient Safety } \\
\text { Culture dimensions }\end{array}$} & \multicolumn{3}{|c|}{ Total Work Experience } & \multirow{3}{*}{$F(\mathrm{df})$} & \multirow{3}{*}{ p-values } \\
\hline & $\begin{array}{c}0-5 \text { years }(1) \\
(\mathrm{n}=81)\end{array}$ & $\begin{array}{c}6-10 \text { years }(2) \\
(n=114)\end{array}$ & $\begin{array}{c}\geq 10 \text { years }(3) \\
\quad(n=759)\end{array}$ & & \\
\hline & n/Mean (SD) & n/Mean (SD) & n/Mean (SD) & & \\
\hline \multicolumn{6}{|l|}{ Outcome Measures } \\
\hline $\begin{array}{l}\text { Frequency of } \\
\text { Event Reporting }\end{array}$ & $79 / 3.05(0.83)$ & $113 / 3.19(0.83)$ & $740 / 3.43(0.83)$ & $10.592(2)$ & $3>1(\mathrm{p}=0.000)$ \\
\hline $\begin{array}{l}\text { Overall Perceptions } \\
\text { of Safety }^{2}\end{array}$ & $81 / 3.37(0.79)$ & $114 / 3.45(0.81)$ & $759 / 3.80(0.72)$ & $21.254(2)$ & $\begin{array}{l}3>1(\mathrm{p}=0.000) \\
3>2(\mathrm{p}=0.000)\end{array}$ \\
\hline \multicolumn{6}{|l|}{ Unit Level } \\
\hline $\begin{array}{l}\text { Manager Promoting } \\
\text { safety }^{2}\end{array}$ & $81 / 3.82(0.80)$ & $114 / 3.67(0.79)$ & $758 / 3.82(0.75)$ & $2.144(2)$ & NS \\
\hline Organisational Learning ${ }^{2}$ & $81 / 3.45(0.77)$ & $114 / 3.51(0.61)$ & $758 / 3.64(0.64)$ & $4.280(2)$ & NS \\
\hline $\begin{array}{l}\text { Teamwork Within } \\
\text { Hospital Units }^{2}\end{array}$ & $81 / 4.00(0.61)$ & $114 / 4.02(0.53)$ & $759 / 4.08(0.52)$ & $1.456(2)$ & NS \\
\hline $\begin{array}{l}\text { Communication } \\
\text { Openness }^{1}\end{array}$ & $81 / 3.93(0.61)$ & $114 / 3.85(0.70)$ & $758 / 3.98(0.64)$ & $2.177(2)$ & NS \\
\hline $\begin{array}{l}\text { Feedback and } \\
\text { Communication }^{1}\end{array}$ & $81 / 3.81(0.80)$ & $114 / 3.82(0.73)$ & $758 / 3.95(0.73)$ & $2.834(2)$ & NS \\
\hline $\begin{array}{l}\text { Nonpunitive Response } \\
\text { To Error }^{2}\end{array}$ & $81 / 3.66(0.78)$ & $114 / 3.74(0.78)$ & $759 / 3.83(0.80)$ & $2.208(2)$ & NS \\
\hline Staffing $^{2}$ & $81 / 3.25(0.72)$ & $114 / 3.23(0.66)$ & $759 / 3.53(0.68)$ & $14.354(2)$ & $\begin{array}{l}3>1(\mathrm{p}=0.001) \\
3>2(\mathrm{p}=0.000)\end{array}$ \\
\hline \multicolumn{6}{|l|}{ Hospital Level } \\
\hline $\begin{array}{l}\text { Hospital Management } \\
\text { Support }^{2}\end{array}$ & $80 / 2.70(0.76)$ & $112 / 2.75(0.83)$ & $752 / 3.16(0.83)$ & $21.668(2)$ & $\begin{array}{l}3>1(\mathrm{p}=0.000) \\
3>2(\mathrm{p}=0.000)\end{array}$ \\
\hline $\begin{array}{l}\text { Teamwork Across } \\
\text { Hospital Units }\end{array}$ & $80 / 3.23(0.64)$ & $114 / 3.34(0.63)$ & $754 / 3.51(0.60)$ & $10.194(2)$ & $3>1(p=0.000)$ \\
\hline $\begin{array}{l}\text { Hospital Handoffs } \\
\text { and Transitions }\end{array}$ & $81 / 3.44(0.68)$ & $114 / 3.63(0.62)$ & $746 / 3.61(0.64)$ & $2.821(2)$ & NS \\
\hline \multicolumn{6}{|l|}{$\begin{array}{l}\text { Swedish Added } \\
\text { dimensions }\end{array}$} \\
\hline $\begin{array}{l}\text { Information to } \\
\text { Patient/Relatives }\end{array}$ & $76 / 3.19(0.80)$ & $109 / 3.28(0.81)$ & $714 / 3.45(0.76)$ & $5.491(2)$ & NS \\
\hline Information to Staff ${ }^{2}$ & $79 / 3.50(1.04)$ & $111 / 3.45(0.94)$ & $729 / 3.72(0.82)$ & $6.520(2)$ & $3>2(\mathrm{p}=0.005)$ \\
\hline
\end{tabular}

${ }^{1}$ Response alternative ranging from 1 (Never) to 5 (Always); ${ }^{2}$ Response alternative ranging from 1 (Strongly Disagree) to 5 (Strongly Agree).

culture dimensions and sample characteristics.

\subsection{Methodological Considerations}

Some methodological considerations must be addressed. This study was conducted as a cross-sectional web survey. Data collection was inspired by Dillman [39] to enhance response rate, i.e. that one reminder was sent digitally to all non-respondents after 2 weeks and a second reminder was sent 9 days thereafter. The response rate of $53 \%$ can be seen as average for a web survey [40] and the final sample size of 1023 is regarded as sufficient for testing the overall fit of the regression model, i.e. test of the R2 [41]. In the present study, three different kinds of health care staff were included. This strengthens the generalizability in the present study and also poses a variation in the sample, which may well be consistent with health care staff in other hospital contexts. At the point of data collection, about 2200 (54\%) RNs, 1200 (29\%) ENs and 700 (17\%) physicians were employed at the county council. Of these, 1023 responded to the study (RNs 64\%, ENs 29\% and physicians 7\%), making the distribution of respondents correspond rather well with the eligible sample and with the country as a whole [42].

Internal dropouts varied between $2 \%(n=22)$ for "Manager Promoting Safety" and 31\% $(n=322)$ for "Information to Patient/Relatives". The dimensions put in the beginning of the instrument had fewer dropouts than those towards the end, which might reflect that respondents became weary by answering many previous questions e.g. Clancy \& Wachsler [43]. Nine dimensions had an alpha value between 0.80 and 0.86 , which is to be considered as acceptable to good [44]. The dimension 
Table 6. Contribution of Swedish Hospital Survey on Patient Safety Culture (S-HSOPS) dimensions and sample characteristics on "Overall Perceptions of Safety" and "Frequency of Incident Reporting".

\begin{tabular}{|c|c|c|c|c|c|c|c|c|c|c|}
\hline & \multicolumn{5}{|c|}{ Overall Perceptions of Safety } & \multicolumn{5}{|c|}{ Frequency of Incident Reporting } \\
\hline & $\mathrm{B}$ & SE & $\beta$ & $\mathrm{t}$ & $\mathrm{p}$ & $\mathrm{B}$ & SE & $\beta$ & $\mathrm{t}$ & $\mathrm{p}$ \\
\hline \multicolumn{11}{|l|}{$\begin{array}{l}\text { Patient Safety } \\
\text { Culture dimensions }\end{array}$} \\
\hline \multicolumn{11}{|l|}{ Unit Level } \\
\hline $\begin{array}{l}\text { Manager Promoting } \\
\text { Safety }\end{array}$ & 0.028 & 0.032 & 0.202 & 0.867 & 0.386 & -0.015 & 0.044 & -0.014 & -0.353 & 0.724 \\
\hline $\begin{array}{l}\text { Organisational } \\
\text { Learning }\end{array}$ & 0.231 & 0.039 & 0.070 & 5.990 & 0.000 & 0.220 & 0.053 & 0.170 & 4.179 & 0.000 \\
\hline $\begin{array}{l}\text { Teamwork Within } \\
\text { Hospital Units }\end{array}$ & 0.098 & 0.042 & 0.017 & 2.319 & 0.021 & -0.035 & 0.058 & -0.022 & -0.604 & 0.546 \\
\hline $\begin{array}{l}\text { Communication } \\
\text { Openness }\end{array}$ & 0.020 & 0.035 & 0.009 & 0.579 & 0.563 & 0.117 & 0.048 & 0.089 & 2.459 & 0.014 \\
\hline $\begin{array}{l}\text { Feedback and } \\
\text { Communication }\end{array}$ & 0.009 & 0.034 & 0.105 & 0.256 & 0.798 & 0.139 & 0.046 & 0.121 & 2.997 & 0.003 \\
\hline $\begin{array}{l}\text { Nonpunitive Response } \\
\text { To Error }\end{array}$ & 0.099 & 0.028 & 0.207 & 3.570 & 0.000 & 0.051 & 0.038 & 0.048 & 1.329 & 0.184 \\
\hline Staffing & 0.226 & 0.031 & 0.145 & 7.302 & 0.000 & -0.057 & 0.042 & -0.046 & -1.331 & 0.183 \\
\hline \multicolumn{11}{|l|}{ Hospital Level } \\
\hline $\begin{array}{l}\text { Hospital Management } \\
\text { Support }\end{array}$ & 0.131 & 0.030 & 0.057 & 4.376 & 0.000 & 0.066 & 0.041 & 0.065 & 1.623 & 0.105 \\
\hline $\begin{array}{l}\text { Teamwork Across } \\
\text { Hospital Units }\end{array}$ & 0.070 & 0.041 & 0.135 & 1.740 & 0.082 & 0.027 & 0.056 & 0.019 & 0.485 & 0.627 \\
\hline $\begin{array}{l}\text { Hospital Handoffs } \\
\text { and Transitions }\end{array}$ & 0.157 & 0.036 & 0.067 & 4.309 & 0.000 & -0.004 & 0.050 & -0.003 & -0.072 & 0.943 \\
\hline \multicolumn{11}{|l|}{$\begin{array}{l}\text { Swedish Added } \\
\text { dimensions }\end{array}$} \\
\hline Information to Patient/Relatives & 0.065 & 0.031 & 0.028 & 2.135 & 0.033 & 0.155 & 0.042 & 0.140 & 3.690 & 0.000 \\
\hline Information to Staff & 0.024 & 0.029 & -0.014 & 0.832 & 0.405 & 0.062 & 0.040 & 0.063 & 1.526 & 0.127 \\
\hline \multicolumn{11}{|l|}{ Sample Characteristics } \\
\hline Registered Nurse & \multicolumn{10}{|c|}{ reference group } \\
\hline Enrolled Nurse & -0.023 & 0.045 & -0.046 & -0.510 & 0.610 & 0.133 & 0.062 & 0.070 & 2.147 & 0.032 \\
\hline Physician & -0.133 & 0.082 & -0.002 & -1.623 & 0.105 & -0.229 & 0.113 & -0.069 & -2.025 & 0.043 \\
\hline $\begin{array}{l}\text { Mixed } \\
\text { medical-surgical }\end{array}$ & \multicolumn{10}{|c|}{ reference group } \\
\hline Medical & -0.003 & 0.055 & 0.050 & -0.062 & 0.950 & 0.148 & 0.076 & 0.086 & 1.950 & 0.051 \\
\hline Surgical & 0.077 & 0.056 & -0.040 & 1.392 & 0.164 & 0.067 & 0.077 & 0.038 & 0.869 & 0.385 \\
\hline Sex & -0.085 & 0.058 & 0.071 & -1.462 & 0.144 & 0.096 & 0.079 & 0.041 & 1.219 & 0.223 \\
\hline Managerial function & 0.234 & 0.084 & -0.003 & 2.783 & 0.006 & -0.177 & 0.115 & -0.048 & -1.544 & 0.123 \\
\hline $\begin{array}{l}\text { Total experience } \\
6-10 \text { years }\end{array}$ & \multicolumn{10}{|c|}{ reference group } \\
\hline $\begin{array}{l}\text { Total experience } \\
0-5 \text { years }\end{array}$ & -0.009 & 0.075 & 0.081 & -0.118 & 0.906 & -0.161 & 0.104 & -0.052 & -1.555 & 0.120 \\
\hline $\begin{array}{l}\text { Total experience } \\
>10 \text { years }\end{array}$ & 0.133 & 0.048 & & 2.777 & 0.006 & 0.017 & 0.065 & 0.009 & 0.265 & 0.791 \\
\hline $\mathrm{R}^{2}$ & 0.498 & & & & & 0.273 & & & & \\
\hline Adjusted $\mathrm{R}^{2}$ & 0.486 & & & & & 0.256 & & & & \\
\hline
\end{tabular}


"Frequency of Event Reporting" showed the highest Cronbach's alpha value $(\alpha=0.86)$, which is similar to the value $(\alpha=0.84)$ presented by Sorra $\&$ Nieva [33] for the same dimension. The dimension "Staffing" showed a Cronbach's alpha value of 0.58 , a finding also close to results presented earlier [33] where this dimension showed a value of 0.63 . This dimension was also reported to exhibit similar alpha values in other studies [28, 45-48]. This means that this dimension might consist of vaguely formulated items whose importance for patient safety culture varies due to country and organization. Thus, the results of this dimension must be interpreted with caution.

\subsection{Discussion of Results}

One important finding of this study was that respondents with a managerial function scored higher than nonmanagers on a majority of the dimensions. This finding is consistent with earlier safety culture surveys, where respondents in management positions scored a more positive perception of patient safety culture $[23,49]$. On the other hand, respondents in a non-managerial position scored in ways consistent with a more positive perception of patient safety culture compared to those with a managerial position when it comes to telling others about their mistakes [49]. One explanation for the managers' higher scores in this respect might be that many managers handle patient safety work reports, risk or adverse event reports in accordance with imperative national health care regulations [50], which were introduced in adjacent to the present survey. This might create a feeling of being familiar and up-to date with patient safety work performed at the workplace. These findings may also reflect that managers are more distant from front-line service and direct contact with patients, unaware and unknowing of all situations when the safety of patients is put at risk, which might explain their more optimistic view of patient safety. This difference between managers and non-managerial staff might also mirror a propensity from health care staff to smooth over safety problems when informing managers [23].

This gap in perception of patient safety culture between managers and non-managerial health care staff needs to be bridged. Patient safety is largely created and developed by managers at all levels in an organization [12] and effective management has shown to be important in creating a positive safety environment [51]. Furthermore, nursing leadership has shown positive relations to improved patient outcomes, such as reduced adverse events [52]. When improving patient safety culture, managers need to provide visions of possibilities, of what is right and important and what needs to be done [53], thus managers need to communicate their commitment to patient safety. A starting point could be face-to face meetings with managers and clinical staff where patient safety issues could be discussed. This approach to improving patient safety, inspired by patient safety leadership walk-rounds [54] have shown to be efficient in supporting improved patient safety culture [55].

RNs, ENs and physicians have different views on patient safety culture. ENs scored significantly higher than physicians and RNs for 12 out of 14 dimensions. In a study by Hughes \& Lapane [56], the less educated ENs scored higher than RNs concerning overall resident safety in nursing homes. Similar results were also reported by From et al. [57], where ENs scored higher than RNs regarding the organizational climate and quality of care. These results might be explained by the fact that ENs are more frequently involved in direct care to the hospital patients, thus the scoring represents an assessment of one's own labor and proneness to safety. It may also reflect that RNs are more educated in critical thinking, hence the RNs score lower.

RNs scored higher than physicians, indicating patient safety culture strengths, in three dimensions. One of these dimensions was "Hospital Handoffs and Transitions", regarding transferring patients and information between units and shifts. This result may reflect that RNs have an understanding and knowledge of this aspect of hospital care, since these tasks, e.g. transferring and reporting on patients, as well as documenting patient care, primarily is performed by nurses. Physicians scored higher than RNs in the dimension "Information to Patient/Relatives" regarding situations when an adverse event had happened. The task of informing and managing patients and relatives in connection to adverse events traditionally falls to the physicians, giving them an understanding of the situation. This indicates that the perception of patient safety culture may be related to the context in which the staff operates and have responsibilities. This assumption is supported by earlier findings [58], where the staff with distance to patients and less education scored lowest for patient safety culture. Earlier research on perceptions of patient safety culture also show inconsistencies between RNs and physicians, where RNs scored higher than physicians for most questions [59]. In Haugen et al. [58], variations were reported where nurse anesthetists scored higher than surgeon physicians both in outcome variables as well as for unit level dimensions. Staff having different views on responsibility, influence and participation in development of patient safety has been reported by Cook et al. [60]. These results show that physicians, administrators and nurses considered patient safety as a nurse responsibility, yet only $8 \%$ of the physicians consider nurses as part of the decision-making team [60]. When managers aim at unifying views like the previously mentioned in order to improve patient safety, they need to be visible to front 
line staff [59] and bridge the professional boundaries [61]. Professionals from different disciplines must also cooperate [62] in order to make patient safety a responsibility for all health care staff, regardless of profession.

Analyses showed that staff $>40$ years of age scored patient safety culture higher than younger staff for nine dimensions. These findings are in accordance with earlier studies, showing that respondents in their forties evaluate safety culture more positively than those in their twenties and thirties [29]. There is a possibility that older staff members are more capable of identifying existing structures supporting patient safety culture, which has been pointed out in the context of nursing homes [63].

Respondents with longer total experience within health care scored higher than those with shorter experience, indicating patient safety culture strengths. This is a result in line with El-Jardali et al. [32], where more experienced health care staff scored higher for the patient safety culture dimensions regarding event reporting and overall safety. This might be explained by the fact that the staff with short experience had not adapted to the existing culture, and thus were able to have a more critical attitude to prevailing lack of safety. These results also correspond with findings from earlier studies showing that work experience affects nurses' clinical decisionmaking process [64] and thus the safety of patients.

In this study, staff in the mixed, smaller medical-surgical health care division scored higher than staff in the larger medical and surgical health care divisions for all hospital level dimensions. These dimensions include remote manager support, teamwork with other units, and hospital transitions. The Swedish added dimension regarding information to patients when an adverse event happens was also scored higher by staff in the mixed division. According to the answers given to the questions, this indicates that staff at the mixed medical-surgical health care division to a greater extent perceive that the transverse paths of decision-making are short, communication and cooperation with other hospital units works well, and that the senior managers and hospital manager are perceived to be accessible, engaged in patient safety work and able to communicate the importance of safe behavior. This is supported by findings in earlier studies that claim that larger organizations are more likely to be complex, less responsive to employees' needs and concerns and that they have a weak organisational culture [65]. In earlier studies, respondents at the smaller hospitals $(<100$ beds) scored higher, indicating patient safety culture strengths, for the broad dimension "Overall Safety" covering items such as existing patient safety problems, sacrifice of safety to get more done and whether procedures prevent errors. The collaborative spirit of managers and staff, as well as open, flexible relations are of great importance in a safety culture [12], a culture which seemingly exists in the mixed division in this study.

In the present study, the explained variance of $49 \%$ for "Overall Perception of Safety" is found to be higher than the variance of $32 \%$ reported in Alahmadi [24] and 28\% reported in Ballangrud et al. [21], but somewhat lower than the variance of $54 \%$ reported by Pfeiffer [34]. The explained variance of $26 \%$ for "Frequency of Incident Reporting" is similar to the model reported by Ballangrud et al. [21]. The explained variance in the present study might depend on the use of sample characteristics as additional independent variables. The multiple regression analysis showed that variables significantly contributing to "Overall Perceptions of Safety" were "Organisational Learning", "Nonpunitive Response To Error", "Staffing", "Hospital Management Support" and "Hospital Handoffs and Transitions" and the sample characteristics managerial function and total health care work experience $>10$ years. These findings confirm the findings by other researchers regarding "Nonpunitive Response To Error" and "Staffing" as significant contributors to Overall Perceptions of Safety [34]. Organisational Learning was also found to significantly contribute to Overall Perceptions of Safety [24]. Several variables significantly contribute to "Frequency of Incident Reporting"; "Organisational Learning", "Feedback and Communication" and "Information to Patient/Relatives". "Feedback and Communication" was also found in other studies to significantly contribute to "Frequency of Incident Reporting" [21,34].

In this study, health care staff with employment longer than three months was eligible for participation. Further research could examine patient safety culture from newly employed health care staff's point of view, whose opinion might broaden and deepen the knowledge of patient safety culture in relation to different staff characteristics. A further examination of patient safety and health care quality by exploring the link between patient safety culture and quality of care from patients' perspectives could shed light on how quality of care is perceived by the ultimate recipient of care; the patient.

\subsection{Conclusions and Implications for Nursing}

Results from the present study show that managers perceive patient safety culture to be stronger than nonmanagerial health care staff do and RNs, ENs and physicians have different views of patient safety culture. Patient safety culture also differs with regard to sex, age and total work experience. Results in the present study also indicate that managerial engagement and support as well as an organization's ability to learn from mistakes and thorough information to patients and relatives are positively related to patient safety. Establishing and maintaining a safety culture and learning from mistakes 
to avoid a culture of blame-and-shame in connection to mistakes are also positively related to patient safety. Based on the results, health care is proposed to ensure manager's further engagement in patient safety and to encourage managers to approach health care staff utmost to patients in order to increase their awareness of the actual situation on patient safety culture. Health care staff, regardless of profession, must cooperate with other professionals from different disciplines and clinical settings to make patient safety a responsibility for all. Managers have the responsibility to foster patient safety culture at their workplace and can thus benefit from the results in this study. Bridging the gap in perception of safety, between managers and non-managers and between different health care staff groups in order to improve patient safety is of utmost importance.

\section{ACKNOWLEDGEMENTS}

The authors would like to thank Jari Appelgren for statistical support.

\section{REFERENCES}

[1] Baker, R., Norton, P., Flintoft, V., Blais, R., Brown, A., Cox, J., Etchells, E., Ghali, W., Hébert, P., Majumdar, S., O'Beirne, M., Palaicos-Derflingher, L., Reid, R., Sheps, S. and Tamblyn, R. (2004) The Canadian Adverse Events Study: The incidence of adverse events among hospital patients in Canada. Canadian Medical Association Journal, 170, 1678-1686.

http://dx.doi.org/10.1503/cmaj.1040498

[2] Brennan, T., Leape, L., Laird, N., Hebert, L., Localio, R., Lawthers, A., Newhouse, J., Weiler, P and Hiatt, H. (1991) Incidence of adverse events and negligence in hospitalized patients. Results of the Harvard Medical practice Study I. The New England Journal of Medicine, 324, 370-376.

http://dx.doi.org/10.1056/NEJM199102073240604

[3] Wilson, R.M., Runciman, W.B., Gibberd, R.W., Harrison, B.T., Newby, L. and Hamilton, J.D. (1995) The quality in Australian health care study. The Medical Journal of Australia, 163, 458-471.

[4] Thomas, E., Studdert, D., Burstin, H., Orav, J., Zeena, T., Williams, E., Howard, M., Weiler, P. and Brennan, T. (2000) Incidence and Types of Adverse Events and Negligent Care in Utah and Colorado. Medical Care, 38, 261271.

http://dx.doi.org/10.1097/00005650-200003000-00003

[5] Schiøler, T., Lipczak, H., Pedersen, B.L., Mogensen, T.S., Bech, K.B., Stockmarr, A., Svenning, A. and Frølich, A. (2001) Forekomstenafutilsigtedehændelserpåsygehuse. En retrospektiv gennemgang af journaler. Ugeskrift for Laeger, 163, 5370.

[6] Vincent, C., Neale, G. and Woloshynowych, M. (2001) Adverse events in British hospitals: Preliminary retrospective record review. British Medical Journal, 322, 517-519. http://dx.doi.org/10.1136/bmj.322.7285.517
[7] Davis, P., Lay-Yee, R., Briant, R., Ali, W., Scott, A. and Schug, S. (2002) Adverse events in New Zealand public hospitals I: Occurrence and impact. New Zealand Medical Journal, 115, 271-279.

[8] Soop, M., Fryksmark, U., Köster, M. and Haglund, B. (2009) The incidence of adverse events in Swedish hospitals: A retrospective medical record review study. International Journal for Quality in Health Care, 21, $285-$ 291. http://dx.doi.org/10.1093/intqhe/mzp025

[9] Zegers, M., de Bruijne, M.C., Wagner, C., Hoonhout, L., Waaijman, R., Smits, M., Hout, F., Zwaan, L., Christiaans-Dingelhoff, I., Timmermans, D., Groenewegen, P. and van der Wal, G. (2009) Adverse events and potentially preventable deaths in Dutch hospitals: Results of a retrospective patient record review study. Quality \& Safety in Health Care, 18, 297-302. http://dx.doi.org/10.1136/qshc.2007.025924

[10] Nieva, V. and Sorra, J. (2003) Safety culture assessment: A tool for improving patient safety in healthcare organizations. Quality \& Safety in Health Care, 12, ii17-ii23. http://dx.doi.org/10.1136/qhc.12.suppl_2.ii17

[11] European Union Network for Quality in Health Care (2006) Use of patient safety culture instrument and recommendations. European Network for Quality in Health Care, Office for Quality Indicators.

[12] Sammers, C., Lykens, K., Singh, K., Mains, D. and Lackan, N. (2010) What is patient safety culture? A Review of the Literature. Journal of Nursing Scholarship, 42, 156-165. http://dx.doi.org/10.1111/j.1547-5069.2009.01330.x

[13] Pronovost, P., Weast, B., Rosenstein, B., Sexton, J., Holzmueller, C., Paine, L., Davis, R. and Rubin, H. (2005). Implementing and validating a comprehensive unit-based safety program. Journal of Patient Safety, 1, 33-40. http://dx.doi.org/10.1097/01209203-200503000-00008

[14] Naveh, E., Katz-Navon, T. and Stern, Z. (2006) Readiness to report medical treatment errors. The effect of safety procedures, safety information, and priority of safety. Medical Care, 44, 117-123. http://dx.doi.org/10.1097/01.mlr.0000197035.12311.88

[15] Vogus, T. and Sutcliffe, K. (2007) The impact of safety organizing, trusted leadership, and care pathways on reported medication errors in hospital nursing units. Medical Care, 45, 997-1002. http://dx.doi.org/10.1097/MLR.0b013e318053674f

[16] Mardon, R., Khanna, K., Sorra, J., Dyer, N. and Famolaro, T. (2010). Exploring relationships between hospital patient safety culture and adverse events. Journal of Patient Safety, 6, 226-232.

http://dx.doi.org/10.1097/PTS.0b013e3181fd1a00

[17] WHO Patient Safety Group (2009). Human Factors in Patient Safety-Review of Topics and Tools. Report for Methods and Measures. WHO/IER/PSP/2009.05.

[18] Zwart, D., Langelaan, M., van de Vooren, R., Kuyvenhoven, M., Kalkman, C., Verheij, T. and Wagner, C. (2011) Patient safety culture measurement in general practice. Clinical properties of "SCOPE". BMC Family Practice, 12, 117. http://dx.doi.org/10.1186/1471-2296-12-117 
[19] Hammer, A., Ernstmann, N., Ommen, O., Wirtz, M., Manser, T., Pfeiffer, Y. and Pfaff, H. (2011) Psychometric properties of the Hospital Survey on Patient Safety Culture for hospital management (HSOPS M). BMC Health Services Research, 11, 165. http://dx.doi.org/10.1186/1472-6963-11-165

[20] Snijders, C., Kollen, B., van Lingen, R., Fetter, W. and Molendijk, H. (2009) Which aspects of safety culture predict incident reporting behavior in neonatal intensive care units? A multilevel analysis. Critical Care Medicine, 37, 61-67.

http://dx.doi.org/10.1097/CCM.0b013e31819300e4

[21] Ballangrud, R., Hedelin, B. and Hall-Lord, M.L. (2012) Nurses' perceptions of patient safety climate in intensive care units: A cross-sectional study. Intensive and Critical Care Nursing, 28, 344-54. http://dx.doi.org/10.1016/j.iccn.2012.01.001

[22] El-Jardali, F., Jaafar, M.,Dimassi, H., Jamal, D. and Hamdan, R. (2010) The current state of patient safety culture in Lebanese hospitals: A study at baseline. International Journal for Quality in Health Care, 22, 386-395. http://dx.doi.org/10.1093/intqhc/mzq047

[23] Wagner, L.M., Capezuti, E. and Rice, J.C. (2009) Nurses' perceptions of safety culture in long-term care settings. Journal of Nursing Scholarship, 41, 184-192. http://dx.doi.org/10.1111/j.1547-5069.2009.01270.x

[24] Alahmadi, H.A. (2010) Assessment of patient safety culture in Saudi Arabian hospitals. Quality and Safety in Health Care, 19, 1-5. http://dx.doi.org/10.1136/qshc.2009.033258

[25] Chen, C. and Li, H. (2010) Measuring patient safety culture in Taiwan using the Hospital Survey on Patient Safety Culture (HSOPSC). BMC Health Services Research, 10, 152.

http://dx.doi.org/10.1186/1472-6963-10-152

[26] Hellings, J., Schrooten, W., Klazinga, N. and Vleugels, A. (2007) Challenging patient safety culture: Survey results. International Journal of Health Care Quality Assurance, 20, 620-632. http://dx.doi.org/10.1108/09526860710822752

[27] Arabloo, J., Rezapour, A., Azar, F. and Mobascheri, Y. (2012) Measuring Patient Safety Culture in Iran Using the Hospital Survey on Patient Safety Culture (HSOPS): An Exploration of Survey Reliability and Validity. International Journal of Hospital Research, 1, 15-28.

[28] Sarac, C., Flin, R., Mearns, K. and Jackson, J. (2011) Hospital survey on patient safety culture: Psychometric analysis on a Scottish sample. BMJ Quality and Safety, 20, 842-848.

http://dx.doi.org/10.1136/bmjqs.2010.047720

[29] Kim, J., An, K., Kim, M. and Yoon, S. (2007) Nurses' Perception of error reporting and patient safety culture in Korea. Western Journal of Nursing Research, 29, 827. http://dx.doi.org/10.1177/0193945906297370

[30] de Wet, C., Johnson, P., Mash, R., McConnachie, A. and Bowie, P. (2010) Measuring perceptions of safety climate in primary care: A cross-sectional study. Journal of Evaluation in Clinical Practice, 18, 135-142. http://dx.doi.org/10.1111/j.1365-2753.2010.01537.x
[31] Scherer, D. and Fitzpatrick, J. (2008) Perceptions of patient safety culture among physicians and RNs in the perioperative area. AORN Journal, 87, 163-175. http://dx.doi.org/10.1016/j.aorn.2007.07.003

[32] El-Jardali, F., Dimassi, H., Jamal, D., Jaafar, M. and Hemadeh, N. (2011) Predictors and outcomes of patient safety culture in hospitals. BMC Health Services Research, 11, 45. http://dx.doi.org/10.1186/1472-6963-11-45

[33] Sorra, J. and Nieva, V. (2004) Hospital Survey on Patient Safety Culture. AHRQ Publication.

[34] Pfeiffer, Y. and Manser, T. (2010) Development of the German version of the Hospital Survey on Patient Safety Culture: Dimensionality and psychometric properties. Safety Science, 48, 1452-1462. http://dx.doi.org/10.1016/j.ssci.2010.07.002

[35] The National Board of Health and Welfare (Socialstyrelsen) (2009) To measure patient safety culture: Handbook for patient safety work (Attmätapatientsäkerhetskulturen). The National Board of Health and Welfare, Stockholm.

[36] Nordin, A., Wilde-Larsson, B., Nordström, G. and Theander, K. (2013) Swedish hospital survey on patient safety culture-psychometric properties and health care staff's perception. In Press.

[37] Field, A. (2009) Discovering Statistics Using SPSS. 3rd Edition, SAGE Publications Ltd.

[38] Hair, J., Black, W., Babin, B., Anderson, R. and Tatham, R. (2006) Multivariate data analysis. 6th Edition, Prentice Hall, New Jersey.

[39] Dillman, D.A. (2007) Mail and internet surveys: The tailored design method. 2nd Edition, John Wiley \& Sons Inc., Hoboken.

[40] Baruch Y. (1999) Response rate in academic studies-A comparative analysis. Human Relations, 52, 421-438. http://dx.doi.org/10.1177/001872679905200401

[41] Green S. (1991) How many subjects does it take to do a regression analysis. Multivariate Behavioral Research, 26, 499-510 http://dx.doi.org/10.1207/s15327906mbr2603 7

[42] Swedish Association of Local Authorities and Regions (SALAR) (2009) Statistics about staff employed in county councils. Sveriges Kommuneroch Landsting (SKL). Personalstatistik.

[43] Clancy, K. and Wachsler, R. (1971) Positional effects in shared-cost surveys. The Public Opinion Quarterly, 35, 258-265. http://dx.doi.org/10.1086/267898

[44] George, D. and Mallery, P. (2003) SPSS for windows step by step: A simple guide and reference, 11.0 update. 4th Edition, Allyn \& Bacon, Boston.

[45] Blegen, M., Gearhart, S., O'Brien, R., Sehgal, N. and Allredge, B. (2009) AHRQ's hospital survey on patient safety culture: Psychometric analyses. Journal of Patient Safety, 5, 139-144. http://dx.doi.org/10.1097/PTS.0b013e3181b53f6e

[46] Waterson, P., Griffiths, P., Stride, C., Murphy, J. and Hignett, S. (2010) Psychometric properties of the Hospital Survey on Patient Safety Culture: Findings from the UK. Quality and Safety in Health Care, 19, e2. 
http://dx.doi.org/10.1136/qshc.2008.031625

[47] Smits, M., Christiaans-Dingelhoff, I., Wagner, C., Wal, G. and Groenewegen, P. (2008) The psychometric properties of the "Hospital Survey on Patient Safety Culture" in Dutch hospitals. BMC Health Services Research, 8, 230. http://dx.doi.org/10.1186/1472-6963-8-230

[48] Bodur, S. and Filiz, E. (2010) Validity and reliability of Turkish version of "Hospital Survey on Patient safety Culture" and perception of patient safety in public hospitals in Turkey. BMC Health Services Research, 10, 28. http://dx.doi.org/10.1186/1472-6963-10-28

[49] Singer, S.J., Gaba, D.M., Geppert, J.J., Sinaiko, A.D., Howard, S.K. and Park, K.C. (2003) The culture of safety: Results of an organization-wide survey in 15 California hospitals. BMJ Quality and Safety in Health Care, 12, 112118. http://dx.doi.org/10.1136/qhc.12.2.112

[50] SFS, Government Offices of Sweden. Ministry of Health and Social Affairs. Sveriges Riksdag, Socialdepartementet (2010). Patient Safety Act. Patientsäkerhetslag, 659.

[51] Firth-Cozens, J. and Mowbray, D. (2001) Leadership and the quality of care. Quality in Health Care, 10, ii3-ii7.

[52] Wong, C. and Cummings, G. (2007) The relationship between nursing leadership and patient outcomes: A systematic review. Journal of Nursing Management, 15, 508521. http://dx.doi.org/10.1111/j.1365-2834.2007.00723.x

[53] Zohar, D. and Tenne-Gazit, O. (2008) Transformational leadership and Group Interaction as Climate Antecedents: A social network analysis. Journal of Applied Psychology, 93, 744-757. http://dx.doi.org/10.1037/0021-9010.93.4.744

[54] Frankel, A., Graydon-Baker, E., Neppl, C., Simmonds, T., Gustafson, M. and Gandhi, T. (2003) Patient safety leadership walkrounds. Joint Commission Journal on Quality and Safety, 29, 16-26.

[55] Öhrn, A., Rutberg, H. and Nilsen, P. (2011) Patient safety dialogue: Evaluation of an intervention aimed at achieving an improved patient safety culture. Journal of Patient Safety, 7, 185-192. http://dx.doi.org/10.1097/PTS.0b013e318230e702

[56] Hughes, C. and Lapane, K. (2006) Nurses' and nursing assistants' perceptions of patient safety culture in nursing homes. International Journal for Quality in Health Care, 18, 281-286. http://dx.doi.org/10.1093/intqhe/mzl020
[57] From, I., Nordström, G., Wilde-Larsson, B. and Johansson, I. (2013) Caregivers in older peoples' care: Perception of quality of care, working conditions, competence and personal health. Scandinavian Journal of Caring Sciences, 27, 704-714. http://dx.doi.org/10.1111/j.1471-6712.2012.01083.x

[58] Haugen, A., Søfteland, E., Eide, G., Nortvedt, M., Aase, K. and Harthug, S. (2010) Patient safety in surgical environments: Cross-countries comparison of psychometric properties and results of the Norwegian version of the Hospital Survey on Patient Safety. BMC Health Services Research, 10, 279.

http://dx.doi.org/10.1186/1472-6963-10-279

[59] Pronovost, P., Weast, B., Holzmueller, C., Rosenstein, B., Kidwell, R., Haller, K., Feroli, E., Sexton, J. and Rubin, H. (2003) Evaluation of the culture of safety: Survey of clinicians and managers in an academic medical center. BMJ Quality and Safety in Health Care, 12, 405-410. http://dx.doi.org/10.1136/qhc.12.6.405

[60] Cook, A., Hoas, H., Guttmannova, K. and Joyner, J. (2004) An error by any name. The American Journal of Nursing, 104, 32-43. http://dx.doi.org/10.1097/00000446-200406000-00025

[61] Willman, A. and Friberg, P. (2013) Teamwork \& Improvement Knowledge. Two core competencies for proper and safe care. The Swedish Society of Nursing, The Swedish Society of Medicine, Solna.

[62] Batalden, P. (2010) The leader's work in the improvement of healthcare. Quality and Safety in Health Care, 19, 367-368. http://dx.doi.org/10.1136/qshc.2010.043745

[63] Bonner, A., Castle, N., Men, A. and Handler, S. (2009) Certified nursing assistants' perception of nursing home patient safety culture: Is there a relationship to clinical outcomes? Journal of the American Medical Directors Association, 10, 11-20. http://dx.doi.org/10.1016/j.jamda.2008.06.004

[64] Cho, M. and Jeong, H. (1999) Participation in decisionmaking and expertise of staff nurses. Journal of Korean Community Nursing, 10, 537-548.

[65] Nahavandi, A. and Malekzadeh, A.R. (1993) Organizational culture in the management of mergers. Quorum Books, Westport. 